\title{
ENERGETIC PARTICLE ABUNDANCES AS PROBES OF AN INTERPLANETARY SHOCK WAVE
}

\author{
D. V. Reames \\ Code 661 NASA/Goddard Space Flight Center, Greenbelt, MD 20771 \\ reames@milkyway.gsfc.nasa.gov \\ and \\ A. J. Tylka \\ Code 7652, E. O. Hulburt Center for Space Research, \\ Naval Research Laboratory, Washington, DC 20375 \\ allan.tylka@nrl.navy.mil
}

Submitted to Astrophysical Journal Letters 


\begin{abstract}
We examine the unique abundance variations of $\mathrm{Fe} / \mathrm{O}$ and $\mathrm{He} / \mathrm{H}$ in solar energetic particles from a W09 event of 2001 April 10, that have leaked through the flank of an interplanetary shock launched from W04 on April 9. Shock waves from both events reach the Wind spacecraft on April 11. During the second event, both $\mathrm{Fe} / \mathrm{O}$ and $\mathrm{He} / \mathrm{H}$ begin at low values and rise to maxima near the time of passage of the shock waves, indicating greater scattering for the species with the highest rigidity at a given velocity. Strong modulation of $\mathrm{Fe} / \mathrm{O}$ suggests preferential scattering and trapping of $\mathrm{Fe}$ by the wave spectrum near and behind the intermediate shock. A significant factor may be the residual proton-generated waves from the very hard proton spectrum accelerated by the early shock wave prior to the onset of the second event. Thus, ion abundances from the later event probe the residual wave spectrum at the earlier shock.
\end{abstract}

Subject headings: acceleration of particles - shock waves - Sun: coronal mass ejections (CMEs) - Sun: particle emission - Sun: abundances 


\section{INTRODUCTION}

Recent evidence has shown that solar energetic particles (SEPs) in the large "gradual" SEP events are accelerated at shock waves driven out from the Sun by coronal mass ejections (CMEs) (Kahler et al. 1984; Gosling 1993; Reames 1995, 1999, 2001; Kahler 1994, 2001; Tylka 2001). The acceleration is mediated by proton-amplified Alfvén waves produced as the particles stream away from the shocks (Bell 1978; Lee 1983, 1997). In large SEP events, wave growth also provides an upper bound to observed proton intensities at the "streaming limit" when high wave intensities cause enough particle scattering to throttle the streaming and hence, the growth rate of the waves ( $\mathrm{Ng} \&$ Reames 1994; Reames \& Ng 1998). In the streaming limit, increases in particle and wave intensities near the shock steepen the local spatial gradients with little affect on proton intensities out at $1 \mathrm{AU}$.

Even at a fixed velocity, ions with different charge-to-mass ratios, $Q / A$, resonate with different regions of the wave spectrum, causing complex temporal variations in their abundances (e.g. Tylka, Reames, \& Ng 1999). Different particle species resonate with proton-generated Alfvén waves with wave vector $k=B / \mu P$, where $P$ is the particle's magnetic rigidity and $\mu$ is the cosine of its pitch angle with respect to the magnetic field, $B$. $\mathrm{Ng}$, Reames, \& Tylka (1999) modeled the evolution of the particles and waves in space and time as they propagate from the shock to the observer, successfully reproducing the qualitative behavior that was observed (see also Tylka 2001).

Near the shock, particles are accelerated as they are scattered back and forth across the shock by proton-generated waves. The spectra of ions and waves can approach an equilibrium for which all ions have power-law spectra with a spectral index that depends only on the shock compression ratio (Lee 1983). In this case, the wave spectrum at the shock is always flatter than $k^{-2}$, which is obtained at the maximum shock compression ratio of 4 ; for this spectrum, the scattering mean free path is independent of rigidity. It is generally assumed that the element abundances accelerated by the shock are identical to those of the injected "seed population"; it is further assumed that these abundances do not vary with energy/nucleon or with time. However, as the protons stream away from the shock differences in their velocity and transport can cause their spectrum to flatten and even roll over at low energies. These altered proton spectra generate complex wave spectra, depending upon position and time, through which the heavier ions must pass. Thus, particle abundances probe either the ambient or the self-generated wave spectra away from the shock.

In SEP events that are magnetically well connected to the observer, the initial rapid rise of intensities magnifies differences in the net scattering of different ion species. Species with more scattering will be delayed, by even small changes in their mean pitch angle, and lag other species as their intensities rise. This effect is amplified by comparing the behavior of abundance ratios, such as $\mathrm{Fe} / \mathrm{O}$ or $\mathrm{He} / \mathrm{H}$, at the same velocity. Such ratios will begin at high values and then decline initially when the species in the denominator is scattered more, and conversely. Suppose that all particles are transported through an initial Kolmogorov wave spectrum where the scattering mean free path $\lambda \sim$ 
$P^{1 / 3}$. In this case, $\mathrm{Fe}$ will be scattered less that $\mathrm{O}, \mathrm{Fe}$ will arrive earlier, and $\mathrm{Fe} / \mathrm{O}$ will begin at high values and decrease to a constant value as a function of time. $\mathrm{He} / \mathrm{H}$ will behave similarly.

Reames, Ng, \& Tylka (2000) compared the initial abundance behavior in small and large SEP events. In small events, or in events with soft proton spectra ( $\mathrm{Ng}$, Reames, \& Tylka 2001; Reames 2001), both $\mathrm{Fe} / \mathrm{O}$ and $\mathrm{He} / \mathrm{H}$ declined with time as described for an ambient Kolmogorov wave spectrum above. For intense events, however, Fe/O declined initially while $\mathrm{He} / \mathrm{H}$ rose. This was understood in terms of wave amplification in the large events as follows. If we assume initially that $\mu \sim 1$, then $2 \mathrm{MeV} \mathrm{H}$ will resonate with waves generated by $2 \mathrm{MeV}$ protons, but $\mathrm{He}$ at $2 \mathrm{MeV}^{-1}$ will resonant with waves amplified by protons of twice its velocity, $\sim 8 \mathrm{MeV}$ protons. The $8 \mathrm{MeV}$ protons arrive about an hour earlier, and given sufficiently high intensity and flat spectrum, they will produce waves that preferentially scatter and delay the $\mathrm{He}$, causing $\mathrm{He} / \mathrm{H}$ to rise from an initially depressed value (see Reames et al. 2000; $\mathrm{Ng}$ et al. 2001).

Despite the behavior of $\mathrm{He} / \mathrm{H}$, however, in nearly all of the events we have observed, $\mathrm{Fe} / \mathrm{O}$ at $2.5-10 \mathrm{MeV} \mathrm{amu}^{-1}$ begins at a high value and falls initially. This is not surprising. For $\mu \sim 1,2.5 \mathrm{MeV}$ amu$^{-1} \mathrm{O}^{+6}$ will resonate with waves produced by $18 \mathrm{MeV}$ protons while $\mathrm{Fe}^{+14}$ resonates with waves produced by $\sim 40 \mathrm{MeV}$ protons. It is usually unlikely that the high-energy proton spectra are sufficiently flat and intensities of $40 \mathrm{MeV}$ protons are sufficiently high to grow enough waves in 1-2 hours that cause Fe to scatter more than $\mathrm{O}$. At energies well below $1 \mathrm{MeV} \mathrm{amu}^{-1}$, initially-rising $\mathrm{Fe} / \mathrm{O}$ may be much more common, but above $\sim 2 \mathrm{MeV} a m u^{-1}$ wave generation by protons from the same event cannot explain this behavior.

\section{THE EVENTS OF 2001 APRIL 9 AND 10.}

We consider two SEP events in this paper. Event 1 is associated with an M7.9 Xray event that began at 1520 UT on 2001 April 9, a 2B flare at S21W04, and an $1192 \mathrm{~km}$ $\mathrm{s}^{-1} \mathrm{CME}$ observed at $1554 \mathrm{UT}$. Event 2 is associated with an X2.3 X-ray event that began at 0506 UT on 2001 April 10, a 3B flare at S23W09, and a $2411 \mathrm{~km} \mathrm{~s}^{-1} \mathrm{CME}$ observed at 0530 UT. Both events were observed by the proton detectors on NOAA/GOES as well as those on the Wind and $I M P-8$ spacecraft. Event 1 had peak proton fluxes at $>10 \mathrm{MeV}$ and $>100 \mathrm{MeV}$ of 5 and $0.4\left(\mathrm{~cm}^{2} \mathrm{sr} \mathrm{s}\right)^{-1}$, respectively, a very hard spectrum. For Event 2 the corresponding fluxes were 300 and $0.3\left(\mathrm{~cm}^{2} \mathrm{sr} \mathrm{s}\right)^{-1}$. Shock waves from the two events arrived on April 11 at about 1410 and 1430; their order of association is uncertain.

Figure 1 shows intensities of various ion species and abundance ratios observed during this period on the Wind and IMP-8 spacecraft (see von Rosenvinge et al. 1995). The onset of Event 1 is seen only in the 19-22 MeV protons in this figure; the event is not observed in any of the other ion channels. Event 2 shows a clear onset in all channels and the intensities then rise to peaks after the time of passage of the shocks. Rising abundance ratios are seen in the figure during Event 2 for both $\mathrm{Fe} / \mathrm{O}$ and $\mathrm{He} / \mathrm{H}$. However, the early suppression of $\mathrm{Fe} / \mathrm{O}$ is much stronger at $2.5-3.2 \mathrm{MeV} \mathrm{amu}^{-1}$ than at $5-10 \mathrm{MeV}$ $\mathrm{amu}^{-1}$. 
The evolution of the proton spectra is shown at 2-hr intervals in Figure 2, with the isotropic background prior to Event 1 (shown as a dashed line) subtracted. The spectra during Event 1 are hard but the intensities are quite low. During Event 2 intensities rise a factor of $\sim 100$ near $10 \mathrm{MeV}$, but they decrease slightly above $100 \mathrm{MeV}$.

\section{DISCUSSION AND CONCLUSIONS}

The proton spectra during Event 1 are substantially harder in the $10-60 \mathrm{MeV}$ region, where wave generation might affect the low-energy $\mathrm{Fe} / \mathrm{O}$ ratios, than in the 1998 September 30 event considered by Reames et al. (2000) and $\mathrm{Ng}$ et al. (2001), for example. However, the intensities are a factor of $\sim 50$ lower and, even though the protons from Event 1 have $\sim 15$ hrs to generate waves prior to the arrival of Fe from Event 2, wave generation far beyond the shock of Event 1 is probably minimal.

The key to understanding the suppressed $\mathrm{Fe} / \mathrm{O}$ during the first day of Event 2 may be that these ions must actually leak through the west flank of Shock 1 on their way to Earth during this time. Perhaps the turbulence at this shock, and in the downstream region behind it, scatters and traps $\mathrm{Fe}$ more than $\mathrm{O}$; this strongly suppresses $\mathrm{Fe} / \mathrm{O}$ in those ions that leak through Shock 1 and propagate out toward Earth. However, the process that produces such strong modulation of the 2.5-3.2 $\mathrm{MeV} \mathrm{amu}^{-1} \mathrm{Fe} / \mathrm{O}$ is not fully understood. The suppression of $\mathrm{Fe} / \mathrm{O}$ decreases with increasing energy because of the decreasing spectrum of the protons that generate fewer resonant waves; e.g. waves that affect $\mathrm{Fe}^{+14}$ at $10 \mathrm{MeV} a m u^{-1}$ would be generated by scarce $150 \mathrm{MeV}$ protons. $\mathrm{He} / \mathrm{H}$ is less strongly affected since protons accelerated by Shock 1 in the $5-10 \mathrm{MeV}$ region barely rise above the preexisting isotropic background that provides a seed population.

It is also possible that protons from Event 2 play some role in modifying the turbulence behind Shock 1 , since $\mathrm{Fe} / \mathrm{O}$ is more strongly modulated than $\mathrm{He} / \mathrm{H}$, even when compared at nearly the same rigidities. At a given rigidity, and resonant wave number, $\mathrm{He}$ has half the velocity, and $\mathrm{Fe}$ has $\sim 1 / 4$ the velocity, of $\mathrm{H}$. Therefore, the protons arrive first at a particular location and they have a longer time to modify the wave spectrum to affect $\mathrm{Fe}$ than to affect either He or O. However, Event 2 alone is not responsible for the strong suppression of $\mathrm{Fe} / \mathrm{O}$; there are many events, otherwise similar to Event 2, that show no such suppression. The presence of Shock 1 is an essential ingredient. Unfortunately, the two-shock configuration is too complex for current numerical models to accommodate.

Despite the complexity, the relative abundances of elements with different values of $Q / A$ are powerful tools for probing the spatial and temporal variations in the spectra of interplanetary Alfvén waves that scatter them, especially in the range of $1-10 \mathrm{MeV} \mathrm{amu}^{-1}$. At energies below $\sim 1 \mathrm{MeV} \mathrm{amu}^{-1}$, ion speeds are slow and their abundances are easily affected by a complex spatial pattern of waves generated by $\leq 10 \mathrm{MeV}$ protons that are often copious. Ions with energies above $\sim 10 \mathrm{MeV} a m u^{-1}$ resonate with waves generated by protons of such high-energy that their abundances are rarely influenced by protons from the same event, except in the immediate vicinity of the shock. Variations in $\mathrm{Fe} / \mathrm{O}$ at high energies may also have other origins (e.g. Tylka et al. 2001). 
In the intermediate region from $1-10 \mathrm{MeV} \mathrm{amu}^{-1}$, abundance ratios often respond to wave spectra generated by $1-100 \mathrm{MeV}$ protons from same event, and, on some occasions, their trajectory to Earth traverses a residual wave spectrum left by an earlier event; such is the case presented herein. Another, less dramatic, example of rising $\mathrm{Fe} / \mathrm{O}$ is seen in the 2000 July 14 "Bastille Day" event where, again, the ions must pass through an intervening shock wave (see Reames, Ng \& Tylka 2001). However, the SEP event of 2001 April 10 provides a uniquely clear example of abundance modulation associated with an intermediate interplanetary shock.

The authors would like to thank $\mathrm{C} . \mathrm{K}$. $\mathrm{Ng}$ for helpful discussions and D. Berdichevsky for assistance in identification of the shock waves discussed in this paper. AJT was supported by the NASA Sun-Earth Connection Guest Investigator Program under DPR S13791G and the Office of Naval Research. 


\section{REFERENCES}

Bell, A. R. 1978, Mon. Not. Roy. Astron. Soc., 182, 147.

Gosling, J. T. 1993, J. Geophys. Res., 98, 18949.

Kahler, S. W. 1994, ApJ, 428, 837.

Kahler, S. W. 2001, J. Geophys. Res. 106, 20947.

Kahler, S. W., Sheeley, N. R. Jr., Howard, R. A., Koomen, M. J., Michels, D. J., McGuire, R. E., von Rosenvinge, T. T. \& Reames, D. V. 1984, J. Geophys. Res., 89,9683 .

Lee, M. A. 1983, J. Geophys. Res., 88, 6109.

Lee, M. A. 1997, in: Coronal Mass Ejections, edited by N. Crooker, J. A. Jocelyn, J. Feynman, Geophys. Monograph 99, (AGU press) 227.

Ng, C. K., \& Reames, D. V. 1994 ApJ 424, 1032.

Ng, C. K., Reames, D. V., \& Tylka, A. J. 1999, Geophys. Res. Lett. 26, 2145.

Ng, C. K., Reames, D. V., \& Tylka, A. J. 2001, Proc. $27^{\text {th }}$ Int. Cosmic Ray Conference, Hamburg, 8, 3140 .

Reames, D. V. 1995, Revs. Geophys. (Suppl.) 33, 585.

Reames, D. V. 1999a, Space Sci. Revs., 90, 413.

Reames, D. V. 2001, in Solar and Galactic Composition, Ed. R. F. WimmerSchweingruber, AIP Conf. Proc. 598, p. 153.

Reames, D. V. and Ng, C. K. 1998, ApJ 504, 1002.

Reames, D. V., Ng, C. K., \& Tylka, A. J. 2000, ApJ 531, L83.

Reames, D. V., Ng, C. K., \& Tylka, A. J. 2001, ApJ 548, L233.

Tylka, A. J. 2001, J. Geophys Res. 106, 25333.

Tylka, A. J., Cohen, C. M. S., Deitrich, W. F., Maclennan, C. G., McGuire, R. E., Ng, C. K., \& Reames, D. V. 2001, ApJ 558, L59.

Tylka, A. J., Reames, D. V., \& Ng, C. K. 1999, Geophys. Res. Lett. 26, 2141.

von Rosenvinge, T. T. et al. 1995, Space Sci. Revs. 71, 155. 


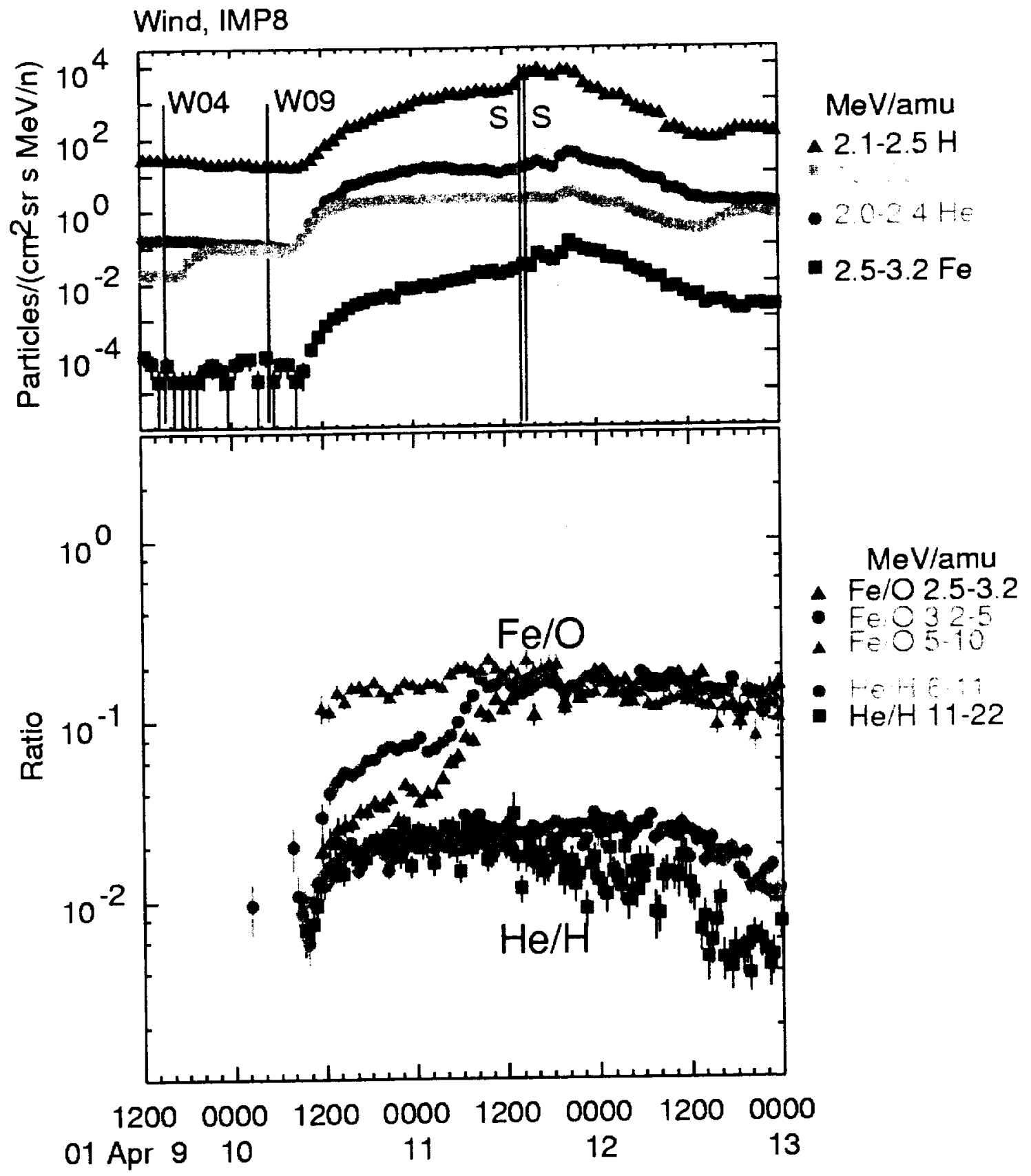

Figure 1. Particle intensities and abundance ratios are shown vs. time during the 2001 April 9-13 period. A particle increase for Event 1, flagged as W04, is seen only in 19-22 $\mathrm{MeV}$ protons. Increases in all particle species are seen for Event 2, flagged as W07. Abundance ratios of both $\mathrm{He} / \mathrm{H}$ and $\mathrm{Fe} / \mathrm{O}$ rise initially in Event 2, and $\mathrm{Fe} / \mathrm{O}$ is strongly suppressed at low energies. 


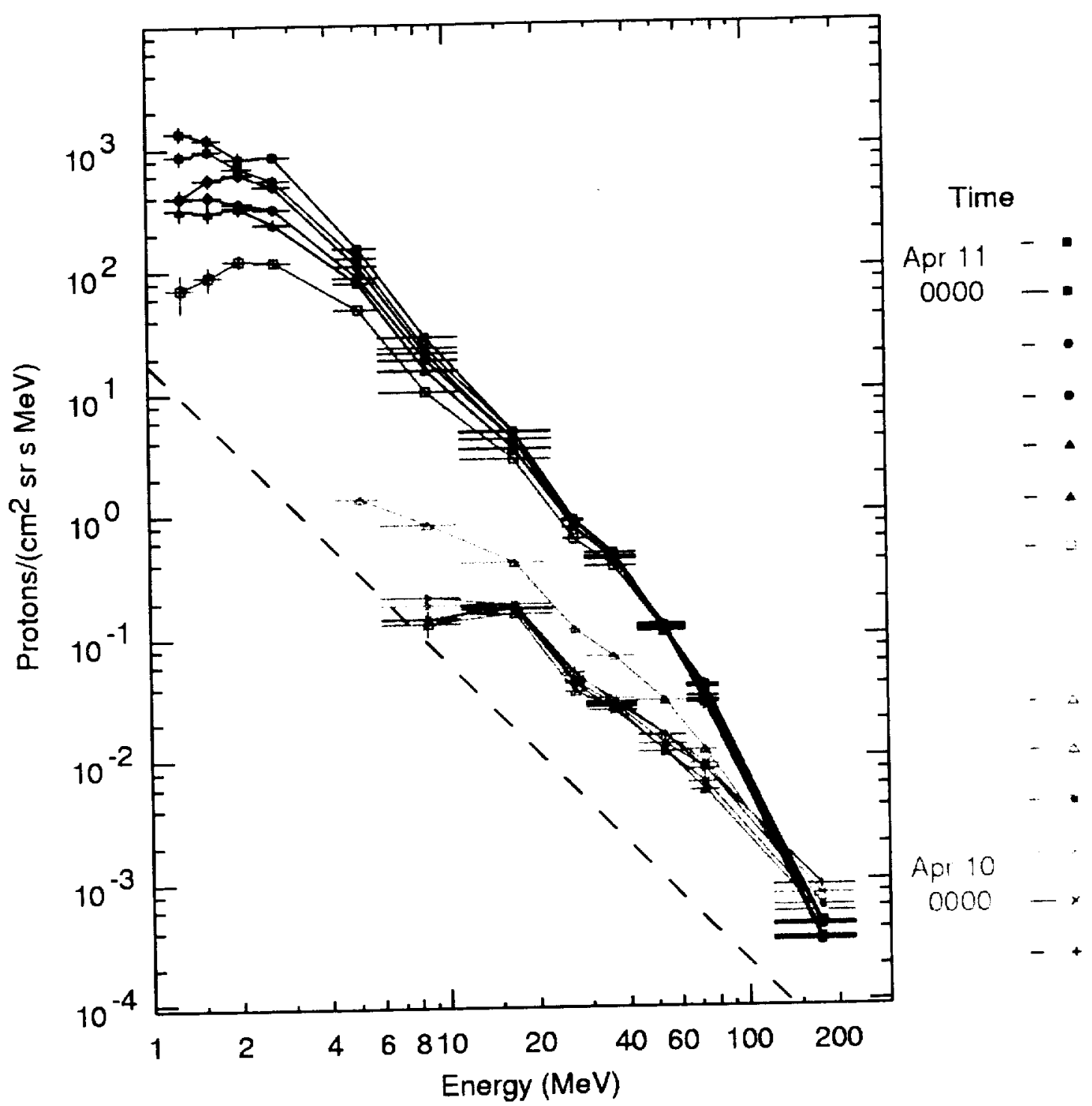

Figure 2. Proton energy spectra are shown at 2-hr intervals from the 2200 on April 9 through 0200 on April 11, contrasting the hard spectra of Event 1 with the softer but more intense spectra during Event 2. Isotropic background prior to Event 1, shown as a dashed line in the figure, has been subtracted from the other spectra. 